Journal of

Molecular Microbiology

and Biotechnology
J Mol Microbiol Biotechnol 2015;25:412-422

DOI: $10.1159 / 000442819$
Received: June 16, 2015

Accepted: November 24, 2015

Published online: January 29, 2016

\title{
The Escherichia coli yjfP Gene Encodes a Carboxylesterase Involved in Sugar Utilization during Diauxie
}

\author{
Nat Johns Algevis Wrench Flavia Loto Ricardo Valladares Graciela Lorca \\ Claudio F. Gonzalez
}

Genetics Institute, Department of Microbiology and Cell Science, Institute of Food and Agriculture Science, University of Florida, Gainesville, Fla., USA

\author{
Key Words \\ Carboxylesterases · Escherichia coli · yjfP · Diauxie · \\ Carbohydrate metabolism
}

\begin{abstract}
Background: Acetylation and efflux of carbohydrates during cellular metabolism is a well-described phenomenon associated with a detoxification process to prevent metabolic congestion. It is still unclear why cells discard important metabolizable energy sources in the form of acetylated compounds. Methods: We describe the purification and characterization of an approximately 28-kDa intracellular carboxylesterase (YjfP) and the analysis of gene and protein expression by qRT-PCR and Western blot. Results: qRT-PCR and Western blot, respectively, showed that yjfP is upregulated during the diauxic lag in cells growing with a mixture of glucose and lactose. The $\beta$-galactosidase activity in the $\triangle y j f P$ strain was both delayed and half the magnitude of that of the wild-type strain. YjfP-hyperproducing strains displayed a long lag phase when cultured with glucose and then challenged to grow with lactose or galactose as the sole carbon source. Conclusion: Our results suggest that YjfP controls the intracellular concentration of acetyl sugars by redirecting them to the main metabolic circuits. Instead of de-
\end{abstract}

toxification, we propose that sugar acetylation is utilized by the cell for protection and to prevent the metabolism of a necessary minimal intracellular sugar pool. Those sugars can eventually be exported as a side effect of these mechanisms.

C 2016 S. Karger AG, Base

\section{Introduction}

A biphasic growth kinetic separated by a short lag phase exhibited by cells growing in the presence of glucose and lactose was described nearly 70 years ago and identified with the name of diauxie [Monod, 1947]. The work of Monod [1947] was an important stepping-stone towards unveiling the induction/repression mechanisms governing the expression of a transcriptional unit they called the Lac operon [Jacob and Monod, 1961]. Since then, the general repression model of the Lac operon has been updated several times and it still constitutes a paradigm for the study of catabolic control of gene expression during diauxie [Kuhlman et al., 2007; Narang and Pilyugin, 2007]. Today, it is accepted that at least 2 more systems in addition to the lac repressor (lacI) regulate the expression of the genes present in the Lac operon (lacZ, $l a c Y$, and $\operatorname{lac} A$ ). Those mechanisms are inducer exclusion

\section{KARGER 125}

(c) 2016 S. Karger AG, Base

$1464-1801 / 16 / 0256-0412 \$ 39.50 / 0$

E-Mail karger@karger.com

www.karger.com $/ \mathrm{mmb}$
Claudio F. Gonzalez

Genetics Institute, Department of Microbiology and Cell Science

University of Florida, 2033 Mowry Rd., Rm. 306

Gainesville, FL 32610-3610 (USA)

E-Mail cfgonzalez@ufl.edu 
[Postma et al., 1993; Saier and Crasnier, 1996] and cAMP catabolic activation [Kimata et al., 1997].

While it is well known that these mechanisms work sequentially during diauxie, they do not explain all of the molecular events that take place during the metabolic transition from glucose to lactose [Stulke and Hillen, 1999]. There are more regulatory secrets still hidden regardless of the 60 years of research on the lac operon. Of particular concern is the biological function of the lacA gene encoding galactoside acetyl transferase (GAT).

It is assumed that this enzyme prevents metabolic congestions by detoxification through sugar acetylation and efflux, but its natural substrate remains unidentified [Andrews and Lin, 1976; Roderick, 2005]. Perhaps our knowledge regarding GAT function has been hindered because $\triangle$ lacA strains do not display a clear phenotype to follow. Nevertheless, sugar acetylation and efflux is a general phenomenon involved in the metabolism of other sugars like maltose. Escherichia coli cells grown in the presence of maltose as the unique carbon source export acetylmaltose to the culture medium. These acetyl sugars cannot be reassimilated and accumulate in the culture fluid [Boos et al., 1981]. The reason for acetylation and export of metabolizable sugars remains to be determined. However, since more than $10 \%$ of intracellular sugars can be in the acetylated form [Wilson and Kashket, 1969] and several cellular systems use the same process, it is assumed to be an important cellular mechanism. It could be used as a detoxification system that presumably mediates the elimination of valuable energy sources in a way that is not detrimental to an organism that exists in a competitive environment and requires that a finely regulated counteractive mechanism be in place.

We hypothesize that, if the acetylated sugars do indeed have a function other than detoxification, their intracellular concentration should be controlled by specific intracellular esterases. Furthermore, these enzymes should be differentially expressed during diauxic growth. It is possible that the significance of sugar acetylation was historically underestimated because of an inability to analyze the general genetic response under such conditions. Transcriptome data have uncovered a higher degree of complexity during diauxic growth [Traxler et al., 2006]. In addition to the regulatory systems described during diauxic transition (diauxic lag), several additional systems work together to coordinate the expression of a subset of genes necessary to adapt the overall cellular physiology to a new carbon source. One primary mechanism of this adaptation is the accumulation of guanosine $3^{\prime}, 5^{\prime}$ bispyrophosphate, a critical signal triggering the general

Carboxylesterase Diauxie Carbohydrate

Metabolism and Acetylation response [Traxler et al., 2006]. However, a large percentage of the differentially regulated proteins detected by transcriptome assays are annotated as hypothetical or even uncharacterized proteins [Pouliot and Karp, 2007]. The lack of evidence about the biological role of proteins differentially expressed impedes rational interpretation of the results previously obtained with microarrays.

To study the regulatory role of esterases during diauxie, we analyzed the published transcriptome data looking for carboxylesterases encoding genes expressed during diauxie. We found that only one gene (yjfP), whose encoded protein displayed a classical esterase motif, was slightly upregulated during diauxie [Traxler et al., 2006]. This protein was previously shown to have esterase activity in screening assays with model substrates [Kuznetsova et al., 2005]. However, the biochemical parameters of the enzyme were not determined and the biological function was not assessed. There is no published evidence associating this protein with any particular biological function, and it has been annotated as a putative thioesterase/ carboxylesterase based only on the activity displayed [Kuznetsova et al., 2005].

In the present work, we describe the biochemical characterization of YjfP, a carboxyl esterase with a preference for short-chain acyl esters as enzymatic substrates. The gene encoding this protein is a single transcription unit induced during diauxic lag, with a lower expression during both exponential phases of growth. E. coli deletion mutants ( $\Delta \mathrm{yjfp}$ ) showed a lower $\beta$-galactosidase activity (compared to the wild type). Furthermore, the metabolism of lactose, maltose, and galactose was impaired in the hyperproducing strain. We hypothesized that YjfP is involved in the hydrolysis of intracellular acetylated sugars and plays a regulatory role during the metabolic adaptation of $E$. coli cells to alternative carbon sources.

\section{Results and Discussion}

\section{Bioinformatic Analysis}

The genes encoding uncharacterized hydrolases differentially expressed on diauxie were selected based on transcriptome data reported by Traxler et al. [2006]. We found that only one gene, i.e. yjfP, whose encoded protein displayed a classical carboxylesterase motif, was slightly upregulated during diauxie. However, the $y j f P$ gene was induced below their cut-off point $\left( \pm 2.5 \log _{2}\right.$-fold). According to the data of Traxler et al. [2006], yjf $P$ was induced during the diauxic lag and immediately repressed once the cells started to utilize lactose. Because the hydrolase encoded by 
Fig. 1. Multiple sequence alignment of YjfP homologues. Conserved residues are shaded black. Similar residues are shade grey. Arrows indicate predicted $\beta$-sheets and rectangles indicate predicted $\alpha$-helices. Dashed-line rectangles indicate regions of homology containing the amino acids that can take part of the catalytic triad. $\mathrm{Eco}=E$. coli; Sdy = Shigella dysenteriae ; Sen = Salmonella enterica; Cko $=C$. koseri; Ent $=$ Enterobacter spp.; Kpn = K. pneumoniae; $\mathrm{Eca}=$ Erwinia carotovora $;$ Ype $=$ Yersinia pestis; Lmo = Listeria monocytogenes; Car = Carnobacterium; Lsa = Lactobacillus salivarius; $\mathrm{Gth}=$ Geobacillus thermodenitrificans; $\mathrm{Bsu}=$ B. subtilis; Tth $=$ T. thermoph lus (1UFO, PDB code). Conserved clusters are indicated by roman numerals (I-VI).



(For second part of figure see next page.) yjf $P$ was uncharacterized and the expression pattern was in agreement with our hypothesis, we decided to characterize this protein and reanalyze the expression of its gene. yjfP is located at $95.16 \mathrm{~min}$ in the E. coli chromosome and is highly conserved in the Enterobacteriaceae family. The 2 genes upstream, i.e. yjf $\mathrm{N}$ and $y j f O$ (both conserved open reading frames), and the genes downstream, i.e. the ula operon (Lascorbate catabolism), are transcribed divergently to yjfP, suggesting that it is a single 750-bp transcriptional unit. The yjfP gene encodes an uncharacterized protein that is 249 amino acids long, annotated as carboxylesterase/ thioesterase [Kuznetsova et al., 2005].

\section{Analysis of the Protein Sequence and the Predicted Structure}

As expected, a BlastP search showed that YjfP homologues were widely spread in the Enterobacteriaceae (fig. 1). The highest degree of conservation was found in Shigella spp. (98\% identity), Citrobacter koseri (72\%), and Salmonella spp. (72\%); a lower degree of identity was found in Enterobacter spp. (61\%), Klebsiella pneumoniae (56\%), and Yersinia spp. (51\%). No paralogues of YjfP were found in E. coli K-12. Homologues with a $30 \%$ protein identity were found in some firmicutes like Bacillus subtilis (yitV gene), and some species of Lactobacillus. All 


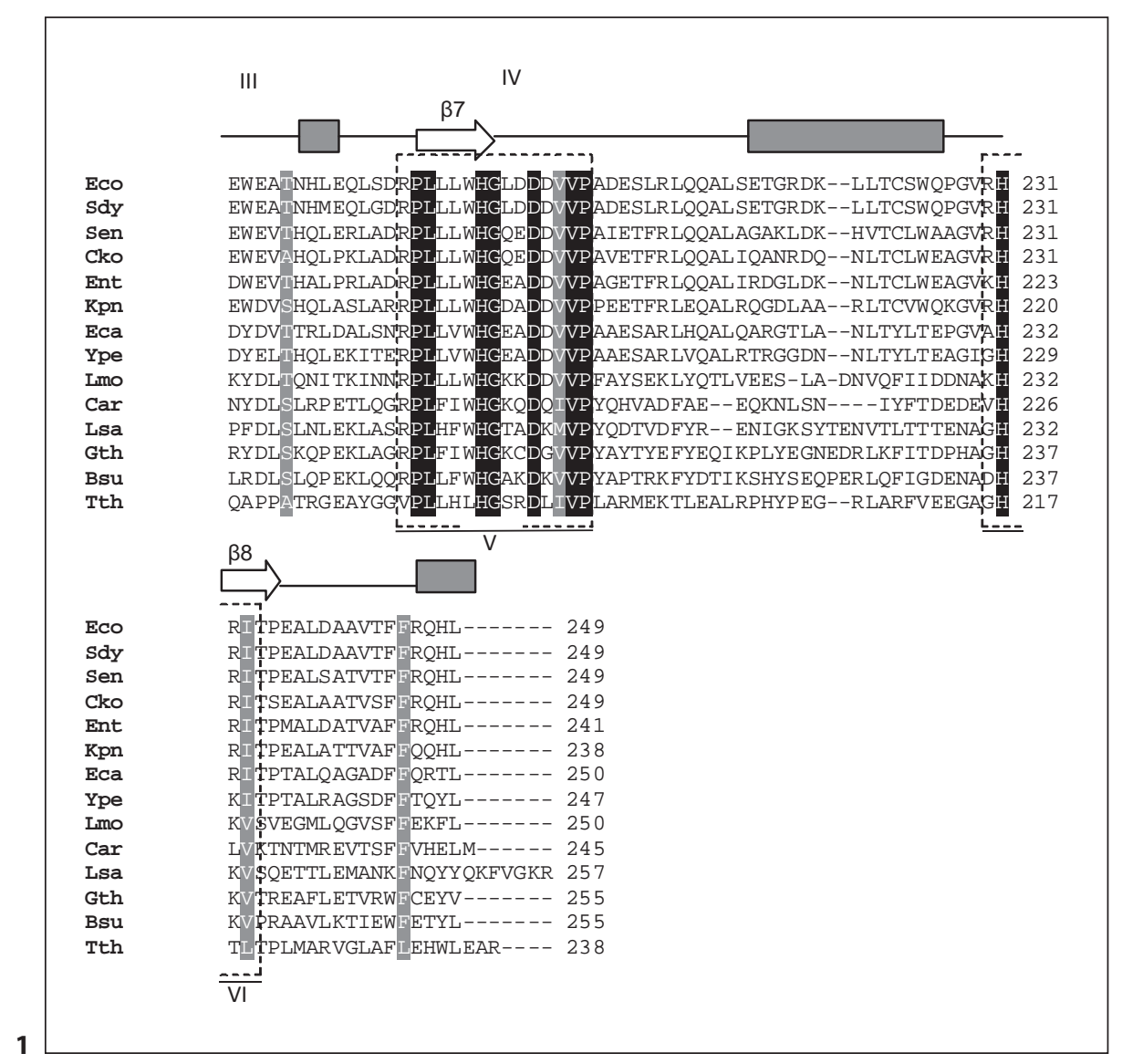

of the proteins retrieved in our BLAST search were annotated as putative esterase proteins. The genes encoding all of these proteins, as well as $y j f P$, are well conserved and not associated with any evident genetic structure suggestive of an operon. The high conservation of a single gene in several groups of bacteria with diverse ecologies suggests a consistent positive evolutionary selection and perhaps a similar biological role.

Analysis of the sequence alignment of this group of proteins showed 6 highly conserved clusters (fig. 1). Typically, the active sites of esterases are formed by 3 conserved amino acids, i.e. serine (usually the nucleophile), an invariable histidine, and an acidic residue aspartate/ glutamic acid [Brenner, 1988]. Cluster III bears the classical serine hydrolase catalytic motif (GXSXG) characteristic of serine esterases. While the His motif is not well represented in the full alignment, the highly conserved His231 in the middle of the sequence GVRHRIT (cluster VI) is in an adequate context for the best candidate that participates at the catalytic site [Cygler et al., 1993]. Block $\mathrm{V}$ contains the unique fully conserved aspartate residue
(Asp197), the potential acid member of the triad. According to the predicted structure, YjfP belongs to the $\alpha / \beta$ hydrolase superfamily (Pfam data base). As displayed in the alignment, the proteins have a predicted canonical $\alpha / \beta$ hydrolase fold with $8 \beta$-strands and 5 predominant $\alpha$-helices. The last protein included in the alignment (TT1662) from Thermus thermophilus has a low sequence identity $(25 \%)$, but it is the most similar protein with a solved structure [Murayama et al., 2005]. The T. thermophilus protein PDB-1UFO displays full conservation of the key residues in all of the critical clusters. This structure was used to correlate the conserved clusters identified in the alignment with conformation of the potential catalytic pocket.

Analysis of the 1UFO structure confirmed that Ser113, His 183, and Asp 217 are in close proximity and in the right orientation in the protein's tertiary structure. The high conservation of the critical residues, the structural similarities, and the correct geometry after folding in TT1662 suggest that YjfP is a serine esterase whose catalytic amino acids at the active site are Ser115, His197, and Asp231. 


\section{Protein Purification and Biochemistry}

YjfP was overproduced and the His6 tagged was purified in 1 step by affinity chromatography; $4.2 \mathrm{mg}$ protein/l of culture media were recovered and successfully dialyzed. The protein was maintained at $-80^{\circ} \mathrm{C}$ in the same dialysis buffer ( $25 \mathrm{~mm}$ Tris-HCl buffer, $\mathrm{pH}$ 8.0, amended with $500 \mathrm{mM} \mathrm{NaCl}$ and $1 \mathrm{~mm}$ DTT to improve stability). Under these conditions, the protein was well preserved and active in thawed samples 1 year after the purification date (the loss of activity was $<5 \%$ ). The purified YjfP protein exhibited a single band with a monomeric molecular weight cut-off of approximately $30 \mathrm{kDa}$ on $12.5 \%$ SDSPAGE (fig. 2) and was eluted as a dimer in analytical gel filtration fast protein liquid chromatography (FPLC) assays. The catalytic activity of the purified protein was assessed using model substrates to understand its substrate preferences. The enzymatic activities of carboxylesterase, thioesterase (to verify the results previously reported) [Kuznetsova et al., 2005], and protease were evaluated. No thioesterase activity was detected towards the 12 different substrates assayed (including palmitoyl-CoA, the substrate used by Kuznetsova et al. [2005]). Since the active site of serine proteases is similar to serine esterases, the YjfP proteolytic activity was evaluated; however, no activity was detected. YjfP showed a hydrolytic activity towards pnitrophenyl esters and $\alpha$-naphthyl esters. Our results suggested that the enzyme only has carboxylesterase activity towards aryl and carboxylic acid esters. $p \mathrm{NP}$ acetate and $p \mathrm{NP}$ butyrate were used as model substrates to determine the optimal biochemical conditions for performance of assays with this enzyme. YjfP displayed the highest activity at $54-56^{\circ} \mathrm{C}$ in a $\mathrm{pH}$ range of 7.6-9.0. Substrate saturation kinetics were performed at $37^{\circ} \mathrm{C}$ and a $\mathrm{pH}$ of 8.6 in $20 \mathrm{~mm}$ CHES buffer; these settings were used to maximize the technique sensitivity and substrate stability. In order to confirm the results of the in silico analysis of the catalytic site, several enzymatic assays were performed in the presence of specific enzymatic inhibitors. Complete inhibition of enzymatic activity was observed in the presence of phenylmethylsulfonylfluoride (PMSF), whereas no significant inhibition was detected with N-ethylmaleimide (NEM; sulfhydryl inhibitor) or EDTA (metal chelator). These results are in accordance with the prediction of a serine (Ser115) as the catalytic nucleophilic amino acid, identified by sequence alignment and predicted via a structure comparison. Divalent cations (used at a final concentration of $1 \mathrm{~mm}$ ) do not stimulate the enzyme, and some inhibition (less than 5\%) was observed in the presence of $\mathrm{Zn}^{2+}$. In the presence of $\mathrm{Cd}^{2+}$ or $\mathrm{Co}^{2+}$, the enzyme displayed $30 \%$ less activity. The

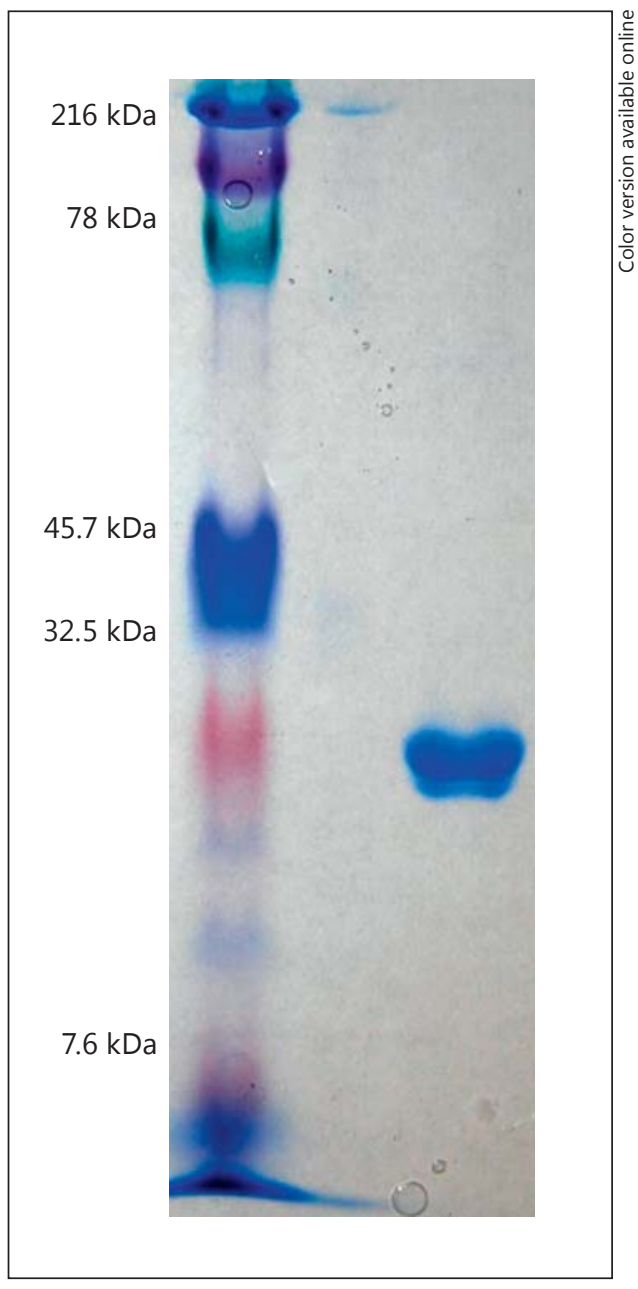

Fig. 2. SDS-PAGE (12\%) of purified YjfP. Lane 1: Kaleidoscope prestained standards; lane 2: $5 \mu \mathrm{g}$ purified YjfP. The molecular weights of the standards are listed between the lanes. The theoretical molecular weight of YjfP (with an $\mathrm{His}_{6}$ tag) is $28.4 \mathrm{kDa}$.

enzymatic profile performed with several substrates showed that the YjfP activity decreased when the acyl chain length of the substrate was increased. No activity was detected with $p \mathrm{NP}$ caproate (C6:0) or $p \mathrm{NP}$ esters with a longer acyl chain. Steady-state kinetics data showed that YjfP displayed a classical Michaelis-Menten hyperbolic saturation. Aryl esters ( $\alpha$ substituted) were hydrolyzed at high velocities $\left(69-84 \mu \mathrm{mol} \times \mathrm{min}^{-1}\right)$ with the highest catalytic efficiency $\left(\mathrm{k}_{\mathrm{cat}} / \mathrm{K}_{\mathrm{m}} 1.3 \times 10^{5}-1.8 \times 10^{5}\right)$. The enzyme showed 10 -fold lower velocities toward $\beta$-naphthyl esters (data not shown). The assays indicated that YjfP had the greatest affinity for $p \mathrm{NP}$ acetate and $\alpha$-naphthyl butyrate, exhibiting $\mathrm{K}_{\mathrm{m}}$ values of 0.080 and $0.092 \mathrm{mM}$, respectively, and comparable apparent $\mathrm{V}_{\max }$ (table 1). The ability of the enzyme to release acetic acid from an acetylated sugar was 


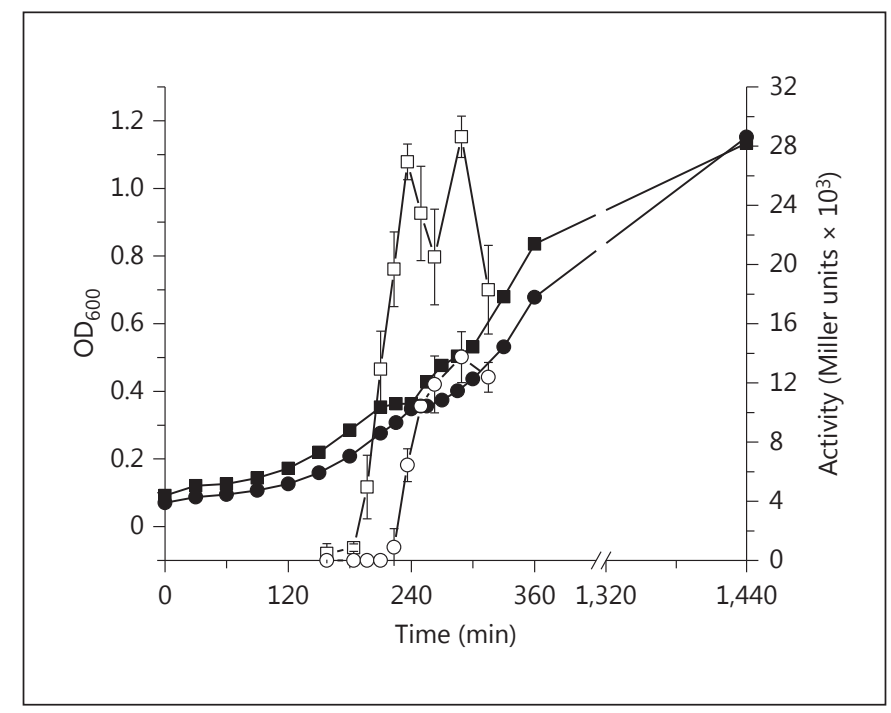

Fig. 3. Diauxie: wild type vs. $\triangle y j f P$. Assays were performed in triplicate in MOPSmm using a mixture of carbon sources, $0.04 \%$ glucose, and $0.2 \%$ lactose. The flasks ( $250-\mathrm{ml}$ volume) containing 25 $\mathrm{ml}$ culture media were inoculated with an initial $\mathrm{OD}_{600}$ of 0.08 0.10 . The cells used as inocula were cultured overnight in MOPSmm with glucose $(0.2 \%)$ as the unique carbon source. Closed symbols represent cellular growth measured as $\mathrm{OD}_{600}$. Closed squares represent the wild-type W3110. Closed circles represent $\Delta y j f P$. Open symbols represent $\beta$-galactosidase activity. Open squares represent the wild-type W3110. Open circles represent $\Delta y j f P$.

Table 1. Kinetics of purified YjfP ( $\left.\mathrm{His}_{6} \mathrm{tag}\right)$

\begin{tabular}{lcccc}
\hline Substrate & $\mathrm{K}_{\mathrm{m}}, \mathrm{mM}$ & $\begin{array}{l}\mathrm{V}_{\max }, \\
\mu \mathrm{mol} \times \mathrm{min}^{-1}\end{array}$ & $\begin{array}{l}\mathrm{k}_{\mathrm{cat}}, \\
\mathrm{s}^{-1}\end{array}$ & $\begin{array}{l}\mathrm{k}_{\mathrm{cat}} / \mathrm{K}_{\mathrm{m}} \\
\mathrm{M}^{-1} \times \mathrm{s}^{-1}\end{array}$ \\
\hline a-Naphthyl acetate & $0.30 \pm 0.02$ & $84.3 \pm 2.0$ & 39 & $1.3 \times 10^{5}$ \\
a-Naphthyl propionate & $0.18 \pm 0.01$ & $69.2 \pm 1.0$ & 32 & $1.8 \times 10^{5}$ \\
a-Naphthyl butyrate & $0.09 \pm 0.00$ & $12.3 \pm 0.1$ & 5.3 & $5.8 \times 10^{4}$ \\
pNP acetate & $0.08 \pm 0.01$ & $13.1 \pm 1.0$ & 5.9 & $7.7 \times 10^{4}$ \\
pNP butyrate & $0.36 \pm 0.01$ & $2.8 \pm 0.3$ & 1.3 & $3.6 \times 10^{3}$ \\
\hline
\end{tabular}

Reaction mixtures contain $25 \mathrm{mM}$ CHES ( $\mathrm{pH}$ 8.6), 0.02-2 mM substrate, and $0.14-0.83 \mu \mathrm{g} / \mathrm{ml}$ enzyme. The enzymatic activity was determined spectrophotometrically at $37^{\circ} \mathrm{C}$. Origin 8 software was used for nonlinear curve fitting.

evaluated using 1-O-methyl-2,3,4,6-tetra-O-acetyl- $\alpha-\mathrm{D}-$ galactoside by HPLC. Acetyl sugars are poorly soluble in water, but YjfP was able to work in the presence of DMSO $5 \%$. The enzymatic activity in these conditions was low, but the increasing amount of acetic acid after the purified protein was added to the mixture was consistent with the enzyme activity (online suppl. fig. 1; for all online suppl.

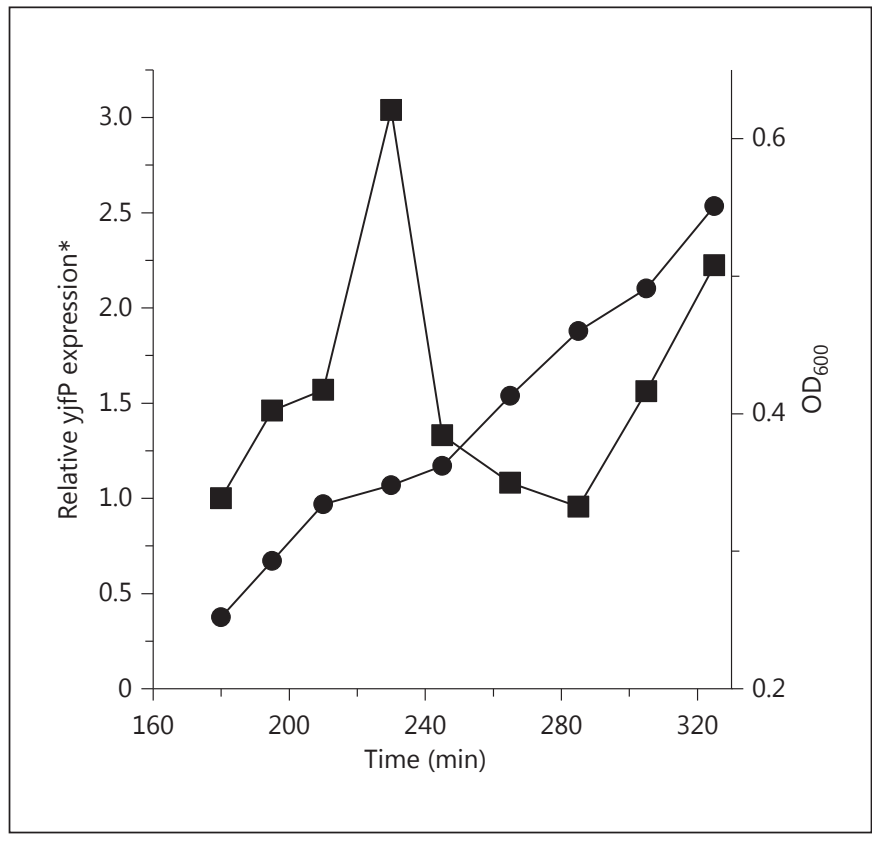

Fig. 4. Expression of yjfP measured during the diauxic lag determined by qRT-PCR. mRNA samples were extracted from WT cells grown in MOPSmm $0.04 \%$ glucose- $0.2 \%$ lactose and processed as described in Methods. The expression of $y j f P$ is indicated relative to the internal standard $r r s C$ amplified in the same run under the same conditions. The diauxic lag started at $210 \mathrm{~min}$ and lasted 30-35 min. Squares represent yjfP expression. Circles indicate $\mathrm{OD}_{600}$ * Expression level relative to the control (control $\left.=1\right)$.

material, see www.karger.com/doi/10.1159/000442819). YjfP demonstrated catalytic flexibility in a range of esterified substrates. The high affinity of the enzyme for acetyl and butyl esters suggested that the natural substrate/s of the enzyme should be esters of a short acyl chain length.

\section{Analysis of Diauxic Growth}

The contribution of YjfP to the cellular physiology of E. coli during diauxie was evaluated by analyzing the cellular growth of W3110 (wild type) and G325KO (knockout mutant) strains. The classical combination of sugars, i.e. glucose $(0.04 \%)$ and lactose $(0.2 \%)$, in MOPS minimal medium (MOPSmm) was used. The cellular growth rate was similar between the 2 strains (apparent specific growth rate $\mu=0.40 \pm 0.05$ ) both before and after diauxic lag. Both strains displayed a typical diauxic lag lasting 25-30 min (fig. 3). The diauxic lag started after $210 \mathrm{~min}$ of culture in the wild-type strain but with a delay of 15-20 min compared to the wild type in G325KO. In both cases, the intracellular $\beta$-galactosidase activity was detected at the beginning of the diauxic lag. The peak of $\beta$-galactosidase ac- 
Fig. 5. Western blots of YjfP-Flag during diauxie. Samples were done using the G325Flag strain, and the strains showed a behavior similar to that of the wild type during growth. The samples were extracted during the diauxic lag, and the relative intensity of the bands was quantified using ImageQuant software.

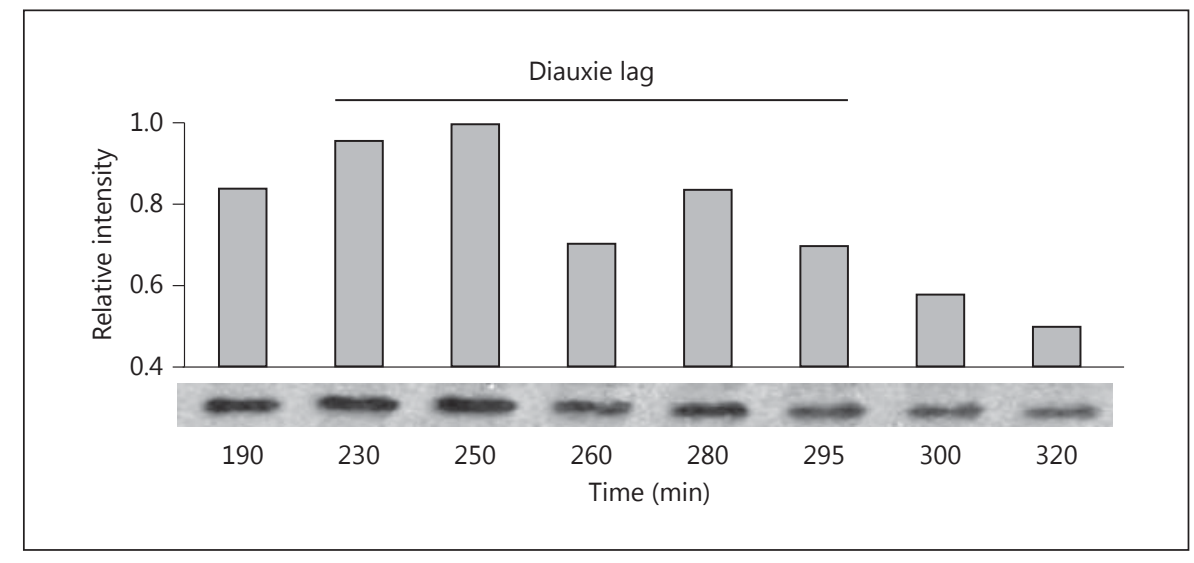

tivity was observed in the middle of the second exponential phase; however, the maximal activity measured in the wild-type W3110 strain was double $(28,000 \pm 2,100$ Miller units) the activity measured in the mutant $(14,000 \pm 1,800$ Miller units; fig. 3). Regardless of the observed difference in $\beta$-galactosidase activity displayed by both strains, it did not have a clear impact on cellular growth rate.

The expression of the $y j f P$ gene was measured by qRTPCR in samples obtained from similar assays; its maximal expression was registered in the middle of the diauxic lag (240 min; fig. 4). The transcript level of $y j f P$ showed a sharp decrease when the cells started the second exponential growth phase. Furthermore, as shown in figure 4, the expression of $y j f P$ increased again when the cells approached the stationary phase of growth. A possible explanation for this phenomenon is that the expression of yjfP could be directly regulated by a stringent response or controlled by starvation sigma factors. The results obtained by qRT-PCR were confirmed by Western blot following the expression of in situ flagged YjfP (fig. 5). Our results confirm that YjfP is induced during the diauxic lag, and in the absence of this carboxylesterase ( $\Delta y j f P$ strain) the intracellular $\beta$-galactosidase activity obtained was significantly lower.

It is well known that cells growing in a mixture of glucose and lactose express the genes encoded in the Lac operon. The last gene in that operon (lacA) encodes a GAT, a functional enzyme with an unknown biological role and an uncertain natural substrate [Roderick, 2005]. The believed function of GAT should be acetylation of the sugars to be exported or, as it can be better expressed, to prevent metabolic congestion [Andrews and Lin, 1976; Fried, 1980]. The results obtained with the $\Delta y j f P$ strain suggest that the enzymatic product of the GAT (acetylated sugars) could be the substrates for the carboxylesterase char- acterized herein. Based on this rationale, the overexpression of YjfP should promote a metabolic gridlock due to sugar toxicity, with a clear impact on cellular growth. To test this hypothesis, we performed several assays using lactose as well as other sugars known to be acetylated and exported to the culture media by E. coli [Andrews and Lin, 1976]. The assays were started with $E$. coli cells cultured overnight on MOPSmm with $0.2 \%$ glucose. In order to ensure full catabolic repression in the cells used as inocula, fresh glucose was added to the overnight culture and the cells were preincubated for $1 \mathrm{~h}$ (see Methods). Furthermore, the cells were inoculated in MOPSmm with glucose, maltose, lactose, or galactose, and growth was monitored by reading the $\mathrm{OD}_{600}$ of each culture. The overproduction of YjfP was performed from the pYjfPM plasmid using $20 \mu \mathrm{M}$ IPTG; the results obtained are depicted in figure 6. The E. coli cells overexpressing YjfP were seriously affected in the presence of either lactose or galactose. An extended lag phase of 6-8 h was observed before the cells started to grow exponentially. When lactose was used, the presence of IPTG, even at low concentrations $(20 \mu \mathrm{M})$, could also stimulate the expression of the lac operon, but it was not enough to overcome the effects YjfP in the W3110Hyp. The cellular growth in the presence of maltose was slightly affected; the lag phase of the W3110Hyp strain was only 30-45 min longer than that of the wild type. Genetic regulation of the genes involved in maltose utilization is far more complex than that of lactose [Dippel and Boos, 2005; Dippel et al., 2005; Reidl and Boos, 1991], and more evidence is necessary to evaluate the impact of YjfP on maltose utilization. Nonspecific toxic effects due to protein overproduction were evident in our assays since control assays using glycerol (nonrepressing conditions) as a carbon source did not have any effect on the cellular growth rate. 
Fig. 6. Growth kinetics of the wild-type strain (W3110) (a) and the YjfP-hyperproducing strain (W3110Hyp) (b) using different sugars as the sole carbon sources. The cells used to inoculate the flasks were cultured overnight in MOPSmm with glucose. Before starting the assays, the cells were preincubated for $1 \mathrm{~h}$ in fresh MOPSmm glucose $(0.2 \%)$ to ensure full catabolic repression. All of the assays were performed in quintuplicate in MOPSmm amended with $20 \mu \mathrm{M}$ IPTG. The cultures were started with equal $\mathrm{OD}_{600}$ of 0.05 and incubated at $37^{\circ} \mathrm{C}$ and $250 \mathrm{rpm}$. Wild-type strain (W3110; closed symbols). Hyperproducer strain (W3110Hyp; open symbols). Squares represent glucose $0.2 \%$. Circles represent lactose $0.2 \%$. Diamonds represent galactose $0.2 \%$. Triangles represent maltose $0.2 \%$.



\section{Conclusion}

Acetylation and efflux of the carbon compounds used as main energy sources by prokaryotic cells are well documented [Shaw, 1967]. However, efflux of valuable energy sources by cells that usually maximize the use of their nutritional resources is difficult to reconcile with the need to compete in a changing environment [Boos et al., 1981; Okamoto and Suzuki, 1965]. The results discussed in this work suggest that acetylation of sugars can play an important role in maximization of the expression of catabolic enzymes ( $\beta$-galactosidase) via a molecular mechanism not yet elucidated. Based on these results, we conclude that the cells maintain a fine balance between the smooth function of the metabolic pathways and the need to maximize the utilization of available carbohydrates. Sugar acetylation could be used by the cell to protect and prevent the metabolism of a minimal intracellular sugar pool. The pool of acetyl sugars can be redirected (recycled) to metabolizable carbohydrates by the intracellular YjfP esterase. By redirecting this intracellular pool, the cells can keep the necessary catabolic genes induced without reliance on sugar uptake (lactose). The acetylated sugars will eventually be exported when the transport rate exceeds the deacylation rate. The catalytic flexibility of the enzyme suggests that YjfP could be active on a broad range of substrates. More evidence will be necessary to evaluate the importance of this protein in regulation of the genes involved in the utilization of other carbon sources.

\section{Methods}

Strain, Plasmids, and Chemicals

The strains and plasmids used in this work are listed in table 2. Bacterial culture stocks were grown in lysogeny broth and maintained in $25 \%$ glycerol at $-80^{\circ} \mathrm{C}$. The antibiotics ampicillin, kanamycin, and chloramphenicol were used in concentrations of 100 , 25 , and $12 \mu \mathrm{g} / \mathrm{ml}$, respectively. All chemicals and reagents were purchased from Sigma or Fisher Scientific unless specified otherwise.

\section{Cloning and Gene Tagging}

The $\Delta y j f p$ mutant was obtained using the $\lambda$ Red recombinase system [Datsenko and Wanner, 2000]. The $\mathrm{Km}^{\mathrm{R}}$ cassette was amplified by PCR using yjfPKnF/R primers. Correct insertion into the chromosome was confirmed by colony PCR using primers upstream and downstream of the yjfP gene ( $y j f P K n F / R c o n f)$.

\section{Epitope Tagging}

In order to follow the protein expression by Western blot, YjfP was tagged at the carboxy terminal end by adding a Flag peptide sequence (DYKDDDDK). The recombinant strain was obtained using the protocol described by Uzzau et al. [2001]. The set of primers used to amplify the $\mathrm{Km}^{\mathrm{R}}$ cassette from the $\mathrm{pKD} 4$ plasmid was $y j f P F l a g F / R$. The primers sequence is described in online supplementary table 1 . 
Table 2. Strains and plasmids

\begin{tabular}{lll}
\hline & Characteristics & References \\
\hline Strains & & \\
W3110 & Wild-type strains [F- $\lambda$-rpos(Am) rph-1 Inv(rrnD-rrnE)] & \\
JW4148 & BW25113 carrying pCAYjfP & Kitagawa et al. [2005] \\
W3110Hyp & Wild-type strain carrying pYjfPM & This work \\
W3110ep & Wild-type strain carrying a pCA24N empty plasmid & This work \\
G325KO & W3110 $\Delta$ yjfP::Kan & This work \\
G325FLAG & W3110 yjfP-FLAG & Datsenko and Wanner [2000] \\
BW25113 & lacI+ $\lambda$ - $\Delta$ lacZ4787(::rrnB-3) hsdR514 $\Delta$ (araBAD)567 & \\
& $\Delta$ (rhaBAD)568 rph-1 & Kitagawa et al. [2005] \\
Plasmids & & Kitagawa et al. [2005] \\
pCA24N & Expression vector, T5 promoter, chloramphenicol resistance \\
pYjfP & pCA24N carrying yjfP ORF & Datsenko and Wanner [2000] \\
pYjfPM & pCA24N carrying yjfP ORF, LacI ${ }^{\mathrm{q}}$ was deleted by inverted PCR & \\
pKD4 & Kanamycin resistance cassette & Datsenko and Wanner [2000] \\
pKD46 & $\lambda$ Red recombinase & \\
\hline
\end{tabular}

\section{Effect of YjfP Overproduction}

The plasmid pCA24N [Kitagawa et al., 2005] was modified for performance of the growth assays and to study the impact of YjfP overproduction on the physiology of $E$. coli. In order to eliminate interferences with the native $E$. coli lactose repression system, the lacI ${ }^{\mathrm{q}}$ repressor encoded in the plasmid was eliminated by inverse PCR [Hemsley et al., 1989].

\section{Protein Overexpression and Purification}

A 4-liter flask containing 2 liters of lysogeny broth and chloramphenicol was inoculated with JW4148 (table 2) from the ASKA collection [Kitagawa et al., 2005] and incubated at $37^{\circ} \mathrm{C}$ and 250 $\mathrm{rpm}$. Upon reaching an $\mathrm{OD}_{600}$ of approximately 0.5 , the culture was incubated on ice for $30 \mathrm{~min}$. Protein overexpression was induced by the addition of IPTG (final concentration $1 \mathrm{mM}$ ), and the culture was incubated overnight at $17^{\circ} \mathrm{C}$ and $250 \mathrm{rpm}$. The remaining steps were carried out at $4^{\circ} \mathrm{C}$ or on ice. The cells were collected by centrifugation for $15 \mathrm{~min}$ at $11,000 \mathrm{rpm}(9,900 \mathrm{~g})$ in a Beckman-Coulter Avanti J-26XP centrifuge. The pellet was resuspended via vortexing in $20 \mathrm{ml}$ binding buffer ( $5 \mathrm{~mm}$ imidazole, $0.5 \mathrm{M} \mathrm{NaCl}$, and $20 \mathrm{~mm}$ Tris- $\mathrm{HCl}, \mathrm{pH} 7.9$ ) and disrupted using a French pressure cell. The disrupted cell suspension was brought up to $30 \mathrm{ml}$ using binding buffer and centrifuged for $20 \mathrm{~min}$ at $19,000 \mathrm{rpm}(29,600 \mathrm{~g})$. The Ni affinity column was prepared as follows: a chromatography column was loaded with $2 \mathrm{ml} \mathrm{His-Bind}$ resin (Novagen), washed 4 times with ultrapure water, and equilibrated with binding buffer. The supernatant described above was applied to the column. The column was washed with 6 volumes of binding buffer and 8 volumes of washing buffer ( $60 \mathrm{~mm}$ imidazole, $0.5 \mathrm{M} \mathrm{NaCl}$, and $20 \mathrm{~mm}$ Tris- $\mathrm{HCl}, \mathrm{pH}$ 7.9). The purified protein was eluted with $10 \mathrm{ml}$ elution buffer ( $1 \mathrm{M}$ imidazole, $0.5 \mathrm{M} \mathrm{NaCl}$, and $20 \mathrm{mM}$ Tris- $\mathrm{HCl}, \mathrm{pH} 7.9$ ) in five $2-\mathrm{ml}$ fractions. The fractions were qualitatively spot-checked with Bradford reagent for the presence of protein and then combined. The protein preparation was dialyzed against 3.5 liters of dialysis/storage buffer $(0.1 \mathrm{M}$ Tris, pH 8, 0.5 M NaCl, and 5 mM DTT) overnight in 10,000 molecular weight cut-off SnakeSkin pleated dialysis tubing (Pierce). The dialyzed protein was aliquoted and stored at $-80^{\circ} \mathrm{C}$ prior to use. A second dialysis was performed later using a 10,000 molecular weight cut-off Slide-W-Lyzer (Pierce) to reduce the DDT concentration. The protein concentration was determined via Bradford assay using a bovine serum albumin (BSA) standard curve [Bradford, 1976]. FPLC (Pharmacia Biotech) analytical gel filtration was performed with an Amersham Superose 12 10/300 GL column (GE Healthcare) using $0.1 \mathrm{M}$ Tris $(\mathrm{pH} 8)$ and $0.5 \mathrm{M} \mathrm{NaCl}$ as a mobile phase. BSA $(67 \mathrm{kDa})$ and $\beta$-lactoalbumin $(35 \mathrm{kDa})$ were used as standards.

\section{Enzymatic Assays}

The carboxylesterase activity was determined spectrophotometrically at $37^{\circ} \mathrm{C}$ with $1-6$ replicates on a UV-1700 PharmaSpec spectrophotometer (Shimadzu) in 1-ml cuvettes or a Synergy HT microplate reader (BioTek) in 96-well plates. Reaction mixtures contained $25 \mathrm{~mm}$ buffer and the appropriate amount of substrate (usually $0.5 \mathrm{mM}$ ). The optimal $\mathrm{pH}$ was determined using the overlapping buffers MES ( $\mathrm{pH}$ 5.4-6.8), HEPES ( $\mathrm{pH} 7.0-8.0$ ), and CHES ( $\mathrm{pH} 8.6-10.0)$ on $p N P$ acetate as the substrate. CHES (pH 8.6) was used as the buffer in subsequent reactions. The model substrates tested were $p \mathrm{NP}$ and $\alpha$ - and $\beta$-naphthyl esters. Stock solutions of $100 \mathrm{mM}$ substrate were prepared with acetonitrile ( $p$ NP esters) and acetone (naphthyl esters). Reactions were initiated by the addition of $0.14-0.83 \mu \mathrm{g} / \mathrm{ml}$ of purified enzyme. Hydrolysis of $p \mathrm{NP}$ esters was detected by $\mathrm{p}$-nitrophenol release and quantified at A410 using the extinction coefficient $\varepsilon=16,300$ $\mathrm{M}^{-1} \cdot \mathrm{cm}^{-1}$. Controls without enzymes were performed to account for self-degradation of $p \mathrm{NP}$ esters in all reactions in which those substrates were used. Naphthyl ester hydrolysis was detected by $\alpha$ - or $\beta$-naphthol release and quantified at A310 in quartz cuvettes with $\varepsilon=3,000 \mathrm{M}^{-1} \cdot \mathrm{cm}^{-1}$. Esterase activity was quantified as micromoles of detected hydrolysis product per minute per microgram of protein. Saturation kinetics were assessed over a range of substrate concentrations, from 0.02 to $2 \mathrm{mM}$. Kinetic parameters $\left(\mathrm{K}_{\mathrm{m}}\right.$ 
and $\mathrm{V}_{\max }$ ) were determined by nonlinear curve fitting using Origin 8 software (Northampton, Mass., USA). Inhibition by EDTA was assessed using 0 and $10 \mathrm{~mm}$ EDTA on $p$ NP butyrate. The effects of divalent cations on the hydrolysis of $p \mathrm{NP}$ butyrate were determined using $1 \mathrm{~mm}$ each of $\mathrm{Fe}^{2+}, \mathrm{Cd}^{2+}, \mathrm{Co}^{2+}, \mathrm{Mn}^{2+}, \mathrm{Ca}^{2+}, \mathrm{Fe}^{3+}, \mathrm{Mg}^{2+}$, $\mathrm{Ni}^{2+}, \mathrm{Cu}^{2+}$, and $\mathrm{Zn}^{2+}$ in the form of chloride salts. The effects of DMSO on the enzymatic activity of $p N P$ acetate were determined at different concentrations of solvent. The effects of the enzymatic inhibitors PMSF and NEM were determined using a 1-mM final concentration of the inhibitor tested.

\section{Carboxyl Esterase Activity towards Acetylated Sugars}

The reaction mixtures for HPLC analysis contained $25 \mathrm{~mm}$ CHES (pH 8.6), 10 mM 1-O-methyl-2,3,4,6-tetra-O-acetyl- $\alpha$-Dgalactoside, DMSO 10\%, and the appropriate amount of YjfPpurified enzyme. Enzymatic digestions were carried out for $4 \mathrm{~h}$ at $37^{\circ} \mathrm{C}$. The reaction mixtures were centrifuged for $10 \mathrm{~min}$ and the supernatants were filtered through 10,000-molecular weight cut-off centrifugal filter tubes (Eppendorf) via centrifugation for $1 \mathrm{~min}$. The samples were prepared for HPLC by acidification with $10 \mu \mathrm{l} 1 \mathrm{~N} \mathrm{H}_{2} \mathrm{SO}_{4}$ and passage through $0.22-\mu \mathrm{m}$ filters. HPLC was performed using a Hewlett-Packard 1090 Series II liquid chromatograph (Agilent) running Hewlett-Packard Chemostation software (Agilent) through an Aminex HPX-87H column (Bio$\mathrm{Rad})$. The solvent was $4 \mathrm{~mm} \mathrm{H}_{2} \mathrm{SO}_{4}$. Acetate detection was achieved using a Hewlett-Packard 1047A refractive index detector (Agilent). The thioesterase activity was assessed using a DTNB [5-(3-carboxy-4-nitrophenyl) disulfanyl-2-nitrobenzoic acid] reduction assay modified from Berge et al. [1981]. The reaction mixture contained $50 \mathrm{~mm}$ HEPES-K, $2 \mathrm{~mm} \mathrm{Na}_{2}$ EDTA, 0.3 $\mathrm{mm}$ DTNB, $40 \mu \mathrm{M}$ thioester substrate, $0.25 \mathrm{mg} / \mathrm{ml} \mathrm{BSA}$, and $5 \mu \mathrm{g}$ purified enzyme. The thioester substrates tested were DL-3-hydroxy-3-methylglutaryl-CoA, methylmalonyl-CoA, phenylacetyl-CoA, hexanoyl-CoA, succinyl-CoA, stearoyl-CoA, octanoylCoA, hydroxybutyryl-CoA, palmitoyl-CoA, methyl-crotonylCoA, glutaryl-CoA, and acetyl-CoA. The thioesterase activity was assessed spectrophotometrically at A412 by measuring the reduction of DTNB by the CoA released by thioester hydrolysis. The peptidase activity was assessed by an azocasein hydrolysis assay [Kilcawley, 2002]. The absorbance was read at $440 \mathrm{~nm}$ in 96-well plates.

For $\beta$-galactosidase, the cells present in the 1-ml culture sample collected from the growth assays were treated with $100 \mu \mathrm{l} 10 \%$ SDS plus $100 \mu \mathrm{l}$ chloroform and vortexed until the cells lysed. Twenty microliters of $2 \%$ CRPG (chlorophenol red- $\beta$-D-galactopyranoside), $20 \mu \mathrm{l}$ lysed cells, and $960 \mu \mathrm{l} \mathrm{Z}$ buffer were combined in an Eppendorf tube. The $\mathrm{Z}$ buffer contained $60 \mathrm{mM} \mathrm{Na}_{2} \mathrm{HPO}_{4}, 40 \mathrm{mM}$ $\mathrm{NaH}_{2} \mathrm{PO}_{4}, 10 \mathrm{mM} \mathrm{KCl}$, and $1 \mathrm{mM} \mathrm{MgSO}_{4}$ and was adjusted to a $\mathrm{pH}$ of 7.0 and stored at $4{ }^{\circ} \mathrm{C}$ until required. Prior to use, $\beta$-mercaptoethanol was added to a final concentration of $50 \mathrm{~mm}$ [Eustice et al., 1991]. The reaction mixture was incubated at $37^{\circ} \mathrm{C}$. Reactions were stopped by the addition of $500 \mu \mathrm{Na}_{2} \mathrm{CO}_{3}$, and the $\mathrm{OD}_{570}$ was measured and the $\beta$-galactosidase activity was expressed in Miller units.

\section{Growth Assays}

The cells used as inocula were cultured overnight aerobically at $37^{\circ} \mathrm{C}$ in MOPSmm [Neidhardt et al., 1974] with $0.2 \%$ glucose as the unique carbon source. Before inoculation, the cultures were diluted with 1 volume of fresh MOPS-glucose $0.2 \%$ and in- cubated for $1 \mathrm{~h}$. This step was done to standardize the inocula and to establish a state of catabolite repression. Growth experiments were performed in $125-\mathrm{ml}$ flasks containing $25 \mathrm{ml}$ culture medium. The flasks were inoculated to $\mathrm{OD}_{600}$ of approximately $0.05-0.1$, and incubated at $37^{\circ} \mathrm{C}$ and $250 \mathrm{rpm}$. Diauxic growth was studied in the same conditions and culture medium but using a mixture of glucose and lactose at a ratio $1: 5(0.04 \%$ glucose $-0.2 \%$ lactose final concentration). During diauxie, the cellular growth was monitored spectrophotometrically at $\mathrm{OD}_{600} \mathrm{ev}-$ ery 15-30 min. Samples were collected as appropriate during readings and stored at $-86^{\circ} \mathrm{C}$. The inocula were made in a similar way when the cells were challenged to growth in the presence of different carbon sources. The IPTG concentration used to induce the expression of YjfP as well as in the control flasks was $20 \mu \mathrm{M}$. In all cases the carbon sources were used at a final concentration of $0.2 \%$ and the flasks were incubated at $37^{\circ} \mathrm{C}$ and $250 \mathrm{rpm}$. All of the growth assays described in the present work were done in quintuplicate.

\section{$q R T-P C R$}

The total RNA of $E$. coli cells collected from the diauxic and conserved at $-80^{\circ} \mathrm{C}$ was isolated using a RiboPure kit (Ambion) in accordance with the manufacturer's instructions and stored at $-80^{\circ} \mathrm{C}$ prior to use. The genetic material extracted was quantified using a NanoDrop 1,000 spectrophotometer. cDNA were synthesized using a SuperScript III First-Strand Synthesis SuperMix for qRT-PCR kit (Invitrogen) in accordance with the manufacturer's instructions and stored at $-20^{\circ} \mathrm{C}$. Quantitative real-time PCR was performed using SYBR GreenER qPCR SuperMix for iCycler (Invitrogen) in a Bio-Rad iCycler iQ (Bio-Rad). The qRT-PCR reaction was performed as previously described [Gonzalez et al., 2006]; yjfPqPCR-F/R were used to amplify an internal sequence of the approximately 200-bp yjfP gene (online suppl. table 1). Amplifications of a similar-length fragment of $\operatorname{rrs} C$ (16S rRNA) with the primers rrsC-F/R were used as standards [Lorca et al., 2007]. The relative expression was calculated as $2^{\text {(CTstandard - CTsample) }}$ [Dietrich et al., 2006].

\section{Western Blot Analysis}

Total protein samples were resolved by SDS-PAGE using $5 \mu \mathrm{g}$ protein per lane. One lane was loaded with purified YjfP (no FLAG) protein as a reference. Following electrophoresis, the lane containing the purified YjPP protein was removed for Coomassie Blue staining. The resolved proteins were transferred to a Nitroplus nitrocellulose membrane (Micron Separations Inc.) by electrophoresis. The processing conditions for the Western blot employed volumes of $50 \mathrm{ml}$ per wash, volumes of $10 \mathrm{ml}$ for the blocking and antibody incubation steps, and rotating agitation in an Isotemp hybridizer (Fisher Scientific) at room temperature. All solutions were made from $0.2 \%(\mathrm{v} / \mathrm{v})$ Tween 20 in phosphate-buffered saline (PBS; $80 \mathrm{mM} \mathrm{Na}_{2} \mathrm{HPO}_{4}, 20 \mathrm{mM}$ $\mathrm{NaH}_{2} \mathrm{PO}_{4}$, and $100 \mathrm{mM} \mathrm{NaCl}, \mathrm{pH} 7.5$ ) - referred to as T-PBS with the appropriate additives. The membrane was blocked with $5 \%$ fat-free dried milk (Nature's First) in T-PBS for $1 \mathrm{~h}$, washed once for $5 \mathrm{~min}$ (all washes were with T-PBS), washed twice for 15 min per wash, incubated overnight in the primary antibody solution (primary antibody diluted 1:3,000, 1\% BSA), washed 4 times for $10 \mathrm{~min}$ per wash, incubated for $1 \mathrm{~h}$ in the secondary antibody solution (secondary antibody diluted 1:10,000), and washed twice for $5 \mathrm{~min}$ at a time, once for $15 \mathrm{~min}$, and twice for 
5 min at a time. The primary antibody was ANTI-FLAG polyclonal affinity-purified rabbit IgG (Sigma). The secondary antibody was Amersham ECL horseradish peroxidase-conjugated anti-rabbit IgG (GE Healthcare). YjfP-FLAG proteins were detected by luminol-based chemiluminescent exposure of BioMax light film (Kodak) using Amersham ECL Western blotting detection reagents (GE Healthcare) in accordance with the manufacturer's instructions.

\section{Acknowledgements}

This work was supported by the Institute of Food and Agricultural Sciences of the University of Florida.

We thank Beverly Driver for her valuable technical assistance. The results presented in this work are part of the undergraduate honors thesis of Nat Johns (University of Florida).

\section{References}

Andrews KJ, Lin EC: Thiogalactoside transacetylase of the lactose operon as an enzyme for detoxification. J Bacteriol 1976;128:510-513.

Berge RK, Hagen LE, Farstad M: Isolation of palmitoyl-CoA hydrolases from human blood platelets. Biochem J 1981;199:639-647.

Boos W, Ferenci T, Shuman HA: Formation and excretion of acetylmaltose after accumulation of maltose in Escherichia coli. J Bacteriol 1981; 146:725-732.

Bradford MM: A rapid and sensitive method for the quantitation of microgram quantities of protein utilizing the principle of protein-dye binding. Anal Biochem 1976;72:248-254.

Brenner S: The molecular evolution of genes and proteins: a tale of two serines. Nature 1988; 334:528-530.

Cygler MJ, Schrag D, Sussman JL, Harel M, Silman I, Gentry MK, Doctor BP: Relationship between sequence conservation and three-dimensional structure in a large family of esterases, lipases, and related proteins. Protein Sci 1993;2:366-382.

Datsenko KA, Wanner BL: One-step inactivation of chromosomal genes in Escherichia coli K-12 using PCR products. Proc Natl Acad Sci USA 2000;97:6640-6645.

Dietrich LEA, Price-Whelan A, Petersen A, Whiteley M, Newman DK: The phenazine pyocyanin is a terminal signalling factor in the quorum sensing network of Pseudomonas aeruginosa. Mol Microbiol 2006;61:1308-1321.

Dippel R, Boos W: The maltodextrin system of Escherichia coli: metabolism and transport. J Bacteriol 2005;187:8322-8331.

Dippel R, Bergmiller T, Bohm A, Boos W: The maltodextrin system of Escherichia coli: glycogen-derived endogenous induction and osmoregulation. J Bacteriol 2005; 187:83328339.

Eustice DC, Feldman PA, Colberg-Poley AM, Buckery RM, Neubauer RH: A sensitive method for the detection of beta-galactosidase in transfected mammalian cells. Biotechniques 1991;11:739-740, 742-743.

Fried VA: lac thiogalactoside transacetylase of Escherichia coli K-12 and ML. J Bacteriol 1980;143:506-509.
Gonzalez CF, Proudfoot M, Brown G, Korniyenko Y, Mori H, Savchenko AV, Yakunin AF: Molecular basis of formaldehyde detoxification: characterization of two S-formylglutathione hydrolases from Escherichia coli, FrmB and YeiG. J Biol Chem 2006;281:1451414522.

Hemsley A, Arnheim N, Toney MD, Cortopassi G, Galas DJ: A simple method for site directed mutagenesis using the polymerase chain reaction. Nucleic Acids Res 1989;17:6545-6551.

Jacob F, Monod J: Genetic regulatory mechanisms in the synthesis of proteins. J Mol Biol 1961;3:318-356.

Kilcawley KN, Wilkinson MG, Fox PF: Determination of key enzyme activities in commercial peptidase and lipase preparations from microbial or animal sources. Enzyme Microb Technol 2002;31:310-320.

Kimata K, Takahashi H, Inada T, Postma P, Aiba $\mathrm{H}$ : cAMP receptor protein-cAMP plays a crucial role in glucose-lactose diauxie by activating the major glucose transporter gene in Escherichia coli. Proc Natl Acad Sci USA 1997; 94:12914-12919.

Kitagawa M, Ara T, Arifuzzaman M, Ioka-Nakamichi $\mathrm{T}$, Inamoto $\mathrm{E}$, Toyonaga $\mathrm{H}$, Mori $\mathrm{H}$ : Complete set of ORF clones of Escherichia coli ASKA library (a complete set of E. coli K-12 ORF archive): unique resources for biological research. DNA Res 2005;12:291-299.

Kuhlman T, Zhang Z, Saier MH Jr, Hwa T: Combinatorial transcriptional control of the lactose operon of Escherichia coli. Proc Natl Acad Sci USA 2007;104:6043-6048.

Kuznetsova EM, Proudfoot M, Sanders SA, Reinking J, Savchenko A, Arrowsmith $\mathrm{CH}$, Edwards AM, Yakunin AF: Enzyme genomics: application of general enzymatic screens to discover new enzymes. FEMS Microbiol Rev 2005;29:263-279.

Lorca GL, Ezersky A, Lunin VV, Walker JK, Altamentova S, Evdokimova E, Vedadi M, Bochkarev A, Savchenko A: Glyoxylate and pyruvate are antagonistic effectors of the Escherichia coli IclR transcriptional regulator. J Biol Chem 2007;282:16476-16491.

Monod J: Studies of the growth of bacterial cultures (in French). Acta Sci Ind 1947;911:1215 .
Murayama K, Shirouzu M, Terada T, Kuramitsu S, Yokoyama S: Crystal structure of TT1662 from Thermus thermophilus HB8: a member of the alpha/beta hydrolase fold enzymes. Proteins 2005;58:982-984.

Narang A, Pilyugin S: Bacterial gene regulation in diauxic and non-diauxic growth. J Theor Biol 2007;244:326-348.

Neidhardt FC, Bloch PL, Smith DF: Culture medium for enterobacteria. J Bacteriol 1974;119: 736-747.

Okamoto S, Suzuki Y: Substrate specificity and kinetic studies on thiogalactoside transacetylase. Biochemistry 1965;12:553-557.

Postma PW, Lengeler JW, Jacobson GR: Phosphoenolpyruvate:carbohydrate phosphotransferase systems of bacteria. Microbiol Rev 1993;57:543-594.

Pouliot Y, Karp PD: A survey of orphan enzyme activities. BMC Bioinformatics 2007;8:244.

Reidl J, Boos W: The malX malY operon of Escherichia coli encodes a novel enzyme II of the phosphotransferase system recognizing glucose and maltose and an enzyme abolishing the endogenous induction of the maltose system. J Bacteriol 1991;173:4862-4876.

Roderick SL: The lac operon galactoside acetyltransferase. C R Biol 2005;328:568-575.

Saier MH Jr, Crasnier M: Inducer exclusion and the regulation of sugar transport. Res Microbiol 1996;147:482-489.

Shaw WV: The enzymatic acetylation of chloramphenicol by extracts of R factor-resistant Escherichia coli. J Biol Chem 1967;242:687-693.

Stulke J, Hillen W: Carbon catabolite repression in bacteria. Curr Opin Microbiol 1999;2:195201.

Traxler MF, Chang DE, Conway T: Guanosine $3^{\prime}, 5^{\prime}$-bispyrophosphate coordinates global gene expression during glucose-lactose diauxie in Escherichia coli. Proc Natl Acad Sci USA 2006;103:2374-2379.

Uzzau S, Figueroa-Bossi N, Rubino S, Bossi L: Epitope tagging of chromosomal genes in Salmonella. Proc Natl Acad Sci USA 2001;98: 15264-15269.

Wilson TH, Kashket ER: Isolation and properties of thiogalactoside transacetylase-negative mutants of Escherichia coli. Biochim Biophys Acta 1969;173:501-508. 